\title{
Switching or sharing in dual-task line-length discrimination?
}

\author{
JEFF MILLER \\ University of Otago, Dunedin, New Zealand \\ and \\ ANNE-MARIE BONNEL \\ Centre National de la Recherche Scientifique, Marseille, France
}

\begin{abstract}
In two experiments, we tested whether subjects switched or shared attention between two simultaneously relevant line-length discrimination tasks. Switching models that allowed within- as well as between-trial switching were considered. In the first experiment, stimulus duration was varied randomly from trial to trial. With varied durations, many switching models predict negative contingencies: for a given duration and attentional allocation, accurate responses on one task should be associated with inaccurate responses on the other task. The results, however, showed no negative contingencies, which is consistent with sharing models. In the second experiment, stimulus duration was reduced to $20 \mathrm{msec}$, yet responses were more than $75 \%$ correct overall. This implies that information was obtained about both of the tasks within single trials, contradicting those switching models which predict that information can be obtained from, at most, one task within a period of $20 \mathrm{msec}$ or less. In short, the results of both experiments support sharing models.
\end{abstract}

A very basic question about the mechanisms subserving spatial attention is: How is attention divided when people try to attend to two locations at the same time? One possibility is that people share attention across locations, simultaneously attending to (i.e., extracting information efficiently from) each location. Another possibility is that they switch attention between locations, extracting information efficiently first from one location and then from another.

Switching models may be further subdivided according to the rate at which attention can be switched. Models in which attention is switched fairly infrequently imply trial-to-trial switching - a strategy in which people attend to one location per experimental trial and switch between locations from one trial to the next (cf. Hughes, 1984; Jonides, 1980). Models with faster switching could permit within-trial switching, in which attention is directed to one location for part of the trial and to another location for the rest of it. Depending on the du-

Portions of this research were reported at the 1990 meeting of the Psychonomic Society in New Orleans and the 1991 meeting of the International Society for Psychophysics in Durham, NC. The authors are grateful to the department of psychology at the University of Oxford for the loan of computers used to carry out some of the simulations reported in connection with Experiment 1. We also thank Allen Osman and Harold Pashler for useful discussions, and Ritske De Jong, Patricia Haden, J. Toby Mordkoff, Eric Ruthruff, and two anonymous reviewers for helpful comments on earlier versions of the manuscript. Correspondence concerning this article should be addressed to J. Miller, Department of Psychology, University of Otago, P.O. Box 56, Dunedin, New Zealand, or A.-M. Bonnel, Laboratoire de Neurosciences Cognitives, C.N.R.S.L.N.F. 1, 31, Chemin Joseph-Aiguier, 13402 Marseille, France. ration of the trial and the time between attentional switches-which might be referred to as the "attentional moment" - these models could allow any number of switches per trial. Indeed, if switching were fast enough (e.g., once per millisecond), these models would effectively mimic sharing models, just as a fast serial computer appears to handle multiple users in parallel (cf. Schweickert \& Boggs, 1984).

The purpose of the present experiments was to find out whether attention is shared or switched in a dual-task divided-attention paradigm requiring two separate linelength comparisons on each trial. This paradigm has previously served as the basis for development of a quantitative psychophysical model of divided attention (e.g., Bonnel \& Miller, 1994; Bonnel, Possamai, \& Schmitt, 1987), and the model has given a good quantitative account of the relation between discrimination performance and attention. As is the case for many psychophysical models, previous descriptions have emphasized the information available for making the psychophysical judgments and deemphasized the information-processing mechanisms responsible for extracting this information. As a result, the model could be formulated either as a sharing model or as a within-trial switching model, and either option would be consistent with previous results. To further develop this model, then, we sought to discriminate between these two distinct theoretical possibilities.

On each trial of the present dual-task paradigm, one pair of lines was presented to the left of fixation and another pair was presented to the right. The subjects had to make two separate discrimination responses, indicat- 
ing for each side whether the pair of lines on that side was the same or different in length. In different conditions, the subjects were instructed to allot different proportions of their attention to the left side (e.g., $80 \%$, $50 \%$, or $20 \%$ ) and to allot the complementary proportion of their attention to the right side. Discrimination accuracy increases with the proportion of attention given to a side (Bonnel \& Miller, 1994; Bonnel et al., 1987), so there is an accuracy tradeoff between attentional conditions (i.e., conditions producing high accuracy on one side yield low accuracy on the other).

The sample-size model (Green \& Luce, 1974; Luce, 1977) has been found to give excellent fits to the observed between-condition tradeoffs. According to this model, the subject accumulates information about the lines on one side by taking perceptual samples from them. Dividing attention between the two sides amounts to dividing a fixed total number of perceptual samples between the lines on one side and the lines on the other side, and samples can be allocated in any desired proportions (e.g., $80 \%$ to the left and $20 \%$ to the right). Within a side, information is combined across samples by averaging, so $d^{\prime}$ increases with the square root of the number of samples (Green \& Swets, 1966). Thus, the model makes two related quantitative predictions concerning the between-condition tradeoff in judgment accuracy, as measured by $d^{\prime 2}$ : (1) the accuracy of judgments on one side should increase linearly with the amount of attention paid to that side, and (2) the total accuracy on the two sides (sum of the $d^{\prime 2}$ values) should remain constant across attentional conditions. Both of these predictions have been verified in experiments requiring concurrent line-length discriminations (Bonnel \& Miller, 1994; Bonnel et al., 1987).

Another finding that is consistent with the samplesize model is that there is no within-condition tradeoff. That is, considering a set of trials from a given attentional condition, the accuracy of one response on a given trial is independent of the accuracy of the other response (i.e., the two responses show zero contingency). This finding is especially important in the present context, because it rules out trial-to-trial switching models. As noted by Sperling and Melchner (1978a, 1978b), trialto-trial switching models predict a negative contingency between the accuracy of the two responses made on the same trial: when the response on one side is correct, the response on the other side is likely to be wrong, and vice versa. The intuition behind this prediction is straightforward. If attention is directed toward one side on any given trial and alternates from side to side between trials, then on any given trial the response should be relatively accurate for the attended side and relatively inaccurate for the other side. Within a condition, then, correct responses on one side should be associated with errors on the other side, and vice versa; that is, there should be a negative contingency between sides, contrary to the observed results. ${ }^{1}$
The sample-size model is consistent with the lack of within-condition tradeoff (i.e., the finding of zero contingency), because the amount of attended processing devoted to a given side (i.e., number of perceptual samples) can be held constant across trials within a condition (cf. Sperling, 1984). According to the model, for example, the subject can allocate $50 \%$ of the perceptual samples to the left side and $50 \%$ to the right side on every trial within a $50 \% / 50 \%$ condition. As long as the amounts of attention (i.e., numbers of samples) devoted to the two sides remain invariant from trial to trial within a condition-rather than fluctuating in a complementary fashion as they do in the trial-to-trial switching modelthe sample-size model need not predict that, within a given attentional condition, an accurate response on one side will be associated with an error on the other.

At issue in the present work is whether invariant allocation of perceptual samples is accomplished by sharing or within-trial switching. The former possibility is that subjects truly share attention across sides - that is, they take samples from both pairs of lines simultaneously, although at slower rates on both sides than would be possible if all attention were allocated to a single side. If subjects can attend to both pairs of lines simultaneously (i.e., share), then they could, in principle, adjust the proportion of attention allocated to each side to match the instructed proportion on every trial within a condition. This would produce the observed performance tradeoffs between conditions, and yet allow the number of samples per side to remain constant across trials within a condition, thereby avoiding within-condition tradeoffs.

The latter possibility is that subjects use a within-trial switching strategy. Following this strategy, they would alternate between pairs quickly enough so that each pair could be attended part of the time on a single trial. For example, they might process the left side for the first $80 \%$ of the available time on each trial and then switch to the right side for the remaining $20 \%$. This strategy would also allow them to get the same number of perceptual samples from a given side on each trial within a condition, thereby avoiding a negative contingency.

Although the notion of within-trial switching may at first seem far-fetched, there is no question that visual attention can be moved rapidly from one location to another, even in the absence of eye movements (e.g., James, 1890). For example, researchers who have examined the movement of attention have provided direct evidence that attention can switch locations rapidly, or perhaps even instantaneously (e.g., Remington \& Pierce, 1984; Shulman, Remington, \& McLean, 1979; Sperling \& Reaves, 1980; Tsal, 1983), although the precise speed of this movement and the mechanism(s) underlying it remain subjects of debate (see Eriksen \& Murphy, 1987). In addition, evidence from visual search tasks suggests that subjects sometimes process display items serially, which implies that attention moves rapidly from one potential target stimulus to the next (e.g., Eriksen, Goettl, 
St. James, \& Fournier, 1989; Estes \& Wessel, 1966; Schneider \& Shiffrin, 1977; Treisman \& Gelade, 1980). Of course, the fact that subjects can switch attention when it is convenient to do so does not imply that this is how they divide attention between locations, but these facts nonetheless imply that within-trial switching must be considered as a possible mechanism for dividing attention across locations. ${ }^{2}$ If subjects can switch attention within a trial, they would have a number of strategies available to allocate attention in accordance with instructions. For example, they might attend to one pair of lines for an initial attended duration, $d_{a} \mathrm{msec}$, and then attend to the other pair for the remainder of the trial, increasing $d_{a}$ with the proportion of attention that they are instructed to allocate to the first pair. Clearly, this strategy would produce an increase in response accuracy with increased attentional emphasis (i.e., betweencondition tradeoffs), but would produce no negative contingency within a condition.

With respect to the goal of discriminating between switching and sharing, a small caveat is in order. We will not be able to distinguish between truly simultaneous sharing and very fast switching (e.g., 50 or more switches per second). In fact, we doubt that this is possible, at least using behavioral data. If switching is very rapid, it can provide an excellent approximation of true sharing, as illustrated by multiuser computer systems. This is only a small caveat, however, because such rapid switching is extremely implausible in the first place. The average time between spikes for an active neocortical neuron is approximately $10-20 \mathrm{msec}$ (Crick \& Asanuma, 1986), and a neuron must be monitored for an interval two or three times this long in order to get an accurate estimate of its firing rate (Marr, 1982; Sejnowski, 1986). Such neural elements would appear to be too slow for rapid switching to be practical, especially given that fine length discriminations may require fairly large populations of neurons to settle into stable states. In addition, studies of attentional movement indicate that under some conditions it is difficult for attention to return to a location for at least $1 \mathrm{sec}$ after leaving it (e.g., Klein, 1988; Maylor \& Hockey, 1985; Posner \& Cohen, 1984). If this "inhibition of return" is operative in the present paradigm, within-trial switching models would be plausible only if they avoided such returns, switching no more than once per trial. In sum, because of the approximate theoretical equivalence of rapid switching and sharing, and because of the a priori implausibility of very rapid switching, we will conclude that we have strengthened the case for sharing to the extent that the data rule out within-trial switching that occurs fairly slowly.

It might seem that previous studies with other tasks would already have settled the question of whether attention is shared or switched, but instead their results suggest that people share attention under some circumstances and switch it under others. For example, Pylyshyn and Storm (1988) found evidence that people shared attention when they were required to monitor a subset of independently moving visual objects, and Hughes (1984) also found evidence for sharing in a location cuing paradigm (see also Jonides, 1980). On the other hand, Sperling and Melchner (1978a, 1978b) found the negative contingencies predicted by trial-to-trial switching models in dual-task visual search, with one task that required detection of a target among a group of inner letters (i.e., displayed near fixation) and another task that required detection of a target among a group of outer letters (i.e., displayed far from fixation). In addition, Shaw $(1980,1982)$ compared performance in divided-attention target-detection tasks against predicted accuracies - but not contingencies-derived from 14 specific sharing and switching models (Shaw, 1982, Table 3). The data supported switching models for some subjects and tasks, but supported sharing models for others. ${ }^{3}$

It is clearly too early to say, in general, which circumstances lead to attentional sharing and which lead to attentional switching. In any case, given the above evidence that attention is sometimes shared and sometimes switched, additional experimentation is clearly required in order to determine whether the sample-size model for dual-task line-length discriminations should be based on a switching or a sharing mechanism.

\section{EXPERIMENTS 1A-1B}

In Experiments 1A-1B, we attempted to discriminate between within-trial switching and true sharing models by using unpredictable stimulus durations. On each trial, the stimulus was presented for one of four durations $(80$, 120,160 , or $280 \mathrm{msec}$ ), randomly intermixed within each block of trials. At the end of the selected duration, the stimulus was followed by a mask, which was designed to terminate perceptual processing.

The basic rationale for this manipulation was that within-trial switching models have difficulty adapting to unpredictable stimulus durations. Consider, for example, how a subject employing a switching mechanism might try to give $50 \%$ of his/her attention to each side. One very reasonable strategy is to attend to the left side for the first half of the trial and then switch to the right side for the second half. As noted earlier, if subjects had used this strategy in previous studies with predictable (i.e., fixed) stimulus durations, they would not have produced negative contingencies, because they would have given the same duration of attended processing to each side on every trial within a condition. This strategy cannot be used with unpredictable stimulus durations, however, because the halfway point cannot be determined until the end of the stimulus, at which point it is too late to make the halftime switch. The exact predictions of switching models depend on the switching strategy that is adopted; a number of specific possibilities are considered below. In general, such models predict negative contingencies (i.e., correct responses concerning one side associated with erroneous responses concerning the other side) within each condition, defined by a certain combination of attentional allocation and stimulus du- 
ration. As discussed below, it is possible for switching models to avoid predicting negative contingencies if their behavior is deterministic, but these models are unattractive because deterministic behavior is implausible a priori, and because they predict unusual patterns of increase in accuracy with increasing stimulus duration.

The predictions of sharing models are more straightforward, on the other hand, because it is not necessary to consider a variety of strategies. Sharing models allow a moment-by-moment division of attention that is insensitive to stimulus duration, so subjects can simply allocate a certain proportion of their capacity to each side and maintain that allocation throughout the trial. Assuming that information is accumulated over the duration of stimulus presentation, this model clearly predicts that performance will increase as a function of stimulus duration. Across trials at a given stimulus duration, the model predicts no contingency between the accuracy of responses on different sides, because the system accumulates the same amount of information about a given side for all trials with that duration. 4

In the following text, we examine the predictions of switching models in more detail. We first consider the predictions of various stochastic switching models (i.e., switching models with trial-to-trial variation in the starting side and/or the duration between switches). We start with trial-to-trial switching, just in case subjects revert to this strategy when faced with uncertain stimulus durations. Next, we consider stochastic models with one switch per trial. Single-switch models seem to be the most plausible within-trial switching models, because they require the fewest switches and because rapid multiswitch models are unlikely a priori, as discussed in the introduction. Nonetheless, we then consider models that allow more than one switch per trial. Although models with very fast within-trial switching are indistinguishable from sharing models, we consider how models with somewhat slower withintrial switching can be distinguished from sharing models. In brief, stochastic switching models of all three kinds (i.e., trial-to-trial, single-switch, and multipleswitch) differ from sharing models in that they predict negative contingencies in an experiment with unpredictable stimulus durations. After considering stochastic switching models, we consider deterministic singleand multiple-switch models. These models are very implausible a priori, because they assume that exactly the same processing occurs on all trials within a condition. We consider them anyway, however, because they do not predict negative contingencies. To provide an exhaustive test of sharing versus switching, then, it is necessary to address the following question: How can sharing models be distinguished from the small and implausible class of switching models that do not predict negative contingencies? Fortunately, the deterministic switching models predict highly distinctive functions relating discrimination accuracy to stimulus duration.

\section{Trial-to-Trial Switching Model}

One strategy is to avoid switching attention within a trial, simply directing it to one side per trial. As in experiments with fixed stimulus durations, this strategy predicts a negative contingency at each stimulus duration (see Sperling \& Melchner, 1978b): The response should be relatively accurate for the side to which attention is directed, and relatively inaccurate for the other side.

\section{Stochastic Single-Switch Model}

According to this model, the subject starts every trial by attending to one side for an initial attended duration $\left(d_{a}\right)$ and then switches attention to the other side for the final $D-d_{a}$ msec, where $D$ is the duration during which the stimulus is available for perceptual analysis. Because the two sides receive attended processing for $d_{a}$ and $D-d_{a}$ msec, respectively, there is a perfect negative correlation (computed across trials within a given stimulus duration) in the amount of attended processing time for the two sides. This negative correlation causes stochastic single-switch models to predict a negative contingency in response accuracy across the two sides, for a given stimulus duration. ${ }^{5}$

\section{Stochastic Multiple-Switch Model}

According to this model, the subject attends to one side at the beginning of every trial and switches sides every $d_{a}$ msec, where $d_{a}$ is a random variable. Regardless of the probability distribution assumed for $d_{a}$, the amount of attended processing time for one side (for a given stimulus duration) is again perfectly negatively correlated with the amount of attended processing time for the other side. ${ }^{6}$ Thus, this model also predicts a negative contingency, within any given condition, in the accuracy of the responses on the two tasks.

\section{Deterministic Single-Switch Model}

This model is similar to the stochastic single-switch model, except that the starting side and the switching time $d_{a}$ are the same on all trials within a condition. Unlike the stochastic switching models, this model predicts that the accuracy of the response to one side will be independent of the accuracy of the response to the other side on the same trial (i.e., zero contingency). The reason for independence is that, at a given stimulus duration, attended processing time does not vary across trials for either side. Since attended processing times on both sides are invariant, they are not negatively correlated; hence, the accuracy of the response concerning one side is independent of the accuracy of the response concerning the other side.

Although this model agrees with the sample-size model in predicting zero contingency, it differs from the sample-size model with respect to its predictions about how accuracy should increase with stimulus duration $D$, as is illustrated in Figure 1. Because the same side is attended first on every trial, this side always receives attended processing for $d_{a} \mathrm{msec}$, regardless of $D$. Thus, 


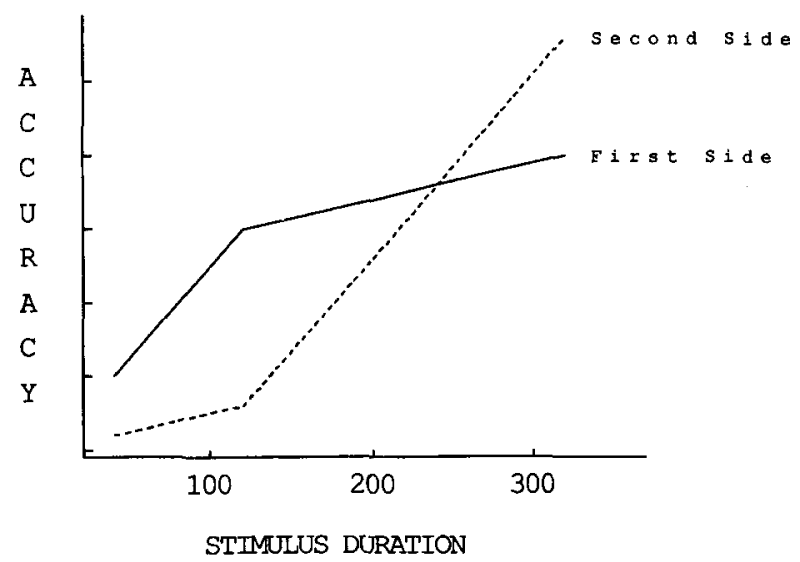

Figure 1. Predicted accuracy (arbitrary axis) as a function of stimulus duration for a deterministic single-switch model in which the subject switches sides after 120 msec.

accuracy on the first-attended side should increase rapidly as $D$ increases to $d_{a}$; meanwhile, accuracy on the second-attended side should increase slowly, if at all, because it gains only increases in unattended processing time. ${ }^{7}$ Once $D$ exceeds $d_{a}$, on the other hand, further increases in $D$ will benefit the second-attended side much more than the first-attended one, because these further increases in $D$ increase the attended processing time for the second side but not the first. In short, this model predicts that any given increment in $D$ ought to increase accuracy on one side much more than on the other. If observed accuracy increases with $D$ at approximately the same rate for both sides, then this model can be rejected.

\section{Deterministic Multiple-Switch Model}

This model is similar to the stochastic multiple-switch model, except that the subject attends to the same side at the beginning of every trial and switches sides every $d_{a}$ msec; the first switch occurs exactly $d_{a}$ msec after stimulus onset (i.e., switches are synchronized to stimulus onset). This procedure is completely deterministic, so it allocates the same amount of attended processing to a given side on every trial with a given stimulus duration. Since the sides do not compete with one another for attention across trials within a condition, zero contingency is predicted.

To the extent that $d_{a}$ is large, however, attended processing time cannot be divided evenly between the two sides. Suppose, for example, that $d_{a}$ is $100 \mathrm{msec}$. On trials with a stimulus duration of $120 \mathrm{msec}$, one side will always receive $100 \mathrm{msec}$ of attended processing, whereas the other side will receive only $20 \mathrm{msec}$. Clearly, responses will be much more accurate for the former side. Furthermore, if stimulus duration is increased to $160 \mathrm{msec}$, the second side should benefit much more than the first one, because attended processing time increases only for the second side.
Figure 2 schematically depicts the constraints on processing accuracy as a function of stimulus duration. Responses to the two sides are equally accurate only for stimulus durations that are a multiple of $2 \times d_{a}$; at all other durations, the first side is performed better. Thus, although this model predicts zero contingencies, like the sharing model, we may be able to discriminate it from the sharing model because of its distinctive predictions about the relation between stimulus duration and accuracy.

\section{Summary}

For a given stimulus duration and attentional allocation, sharing models allow the accuracy of one response to be independent of the accuracy of the other. Stochastic within-trial switching models predict negative contingencies within each of these conditions, because randomized stimulus duration ensures a varying, complementary relationship in the time allocated to the two sides. Deterministic switching models do not predict negative contingencies, but they do predict unequal relations of accuracy to stimulus duration for the two sides. Thus, it should be possible to discriminate between sharing and switching by examining both withincondition contingencies and between-condition relations of accuracy to stimulus duration.

\section{Method}

Apparatus and Stimuli. Subjects were tested in a soundshielded cubicle, with lighting arranged to be as homogeneous as possible. A PDP-12 (Digital Equipment Corp.) computer was programmed to present the stimuli on a cathode ray tube (CRT) and to record responses and response latencies. The CRT was coated with $\mathrm{P} 31$ phosphor, which decays to $1 \%$ in $250 \mu \mathrm{sec}$, and the brightness of the screen was also reduced to minimize persistence. A chinrest was used to maintain a constant distance of $65 \mathrm{~cm}$ between the subject's eyes and the CRT.

The stimuli were two simultaneously presented pairs of vertical lines, with one pair presented on each side of fixation. The inner

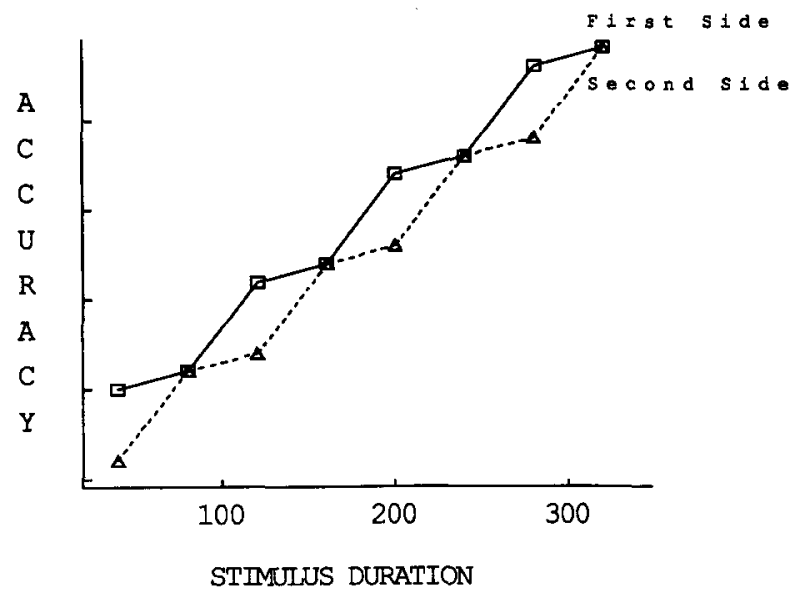

Figure 2. Predicted accuracy (arbitrary axis) as a function of stimulus duration for a deterministic multiple-switch model in which the subject switches sides every $\mathbf{4 0}$ msec. 
and outer lines of each pair were $2.8^{\circ}$ and $3.3^{\circ}$ of visual angle from fixation, respectively. The inner line was always $6.21^{\circ} \mathrm{long}$, with its midpoint horizontally aligned with the fixation point. The outer line was the same length for half of the trials, and it was longer for the other half. The bottoms of all four lines (i.e., both pairs) were always horizontally aligned. Each line was a single pixel (less than $0.1^{\circ}$ ) in width.

Responses were made with the left and right hands on two fivebutton panels, which were assigned to the left and right pairs of stimulus lines, respectively. The buttons under the thumbs and little fingers were used to indicate "same" and "different" responses, respectively. Buttons under the index, middle, and ring fingers were used to indicate high, medium, and low confidence, respectively.

Subjects and Procedure. The subjects were 4 employees of the C.N.R.S. ( 3 male and 1 female), who were paid 40 French francs per hour. All were experienced in dual-task line-length discriminations with unmasked stimuli, and none were aware of the purpose of the study.

Each subject participated in five sessions. Each session included two blocks of 144 trials, separated by a few minutes of rest. Attention was varied between sessions, and the order of attentional conditions was balanced across subjects as much as possible. In each session, a subject was instructed to pay $100 \%, 80 \%, 50 \%$, $20 \%$, or $0 \%$ of his or her attention to the left side, and to pay the complementary percentage of attention to the right side. In the blocks with $100 \%$ attention paid to one side, the stimuli were presented on the unattended side; the subjects were instructed to respond to them, but to pay them as little attention as possible.

The 144 trials within one block consisted of 9 trials in each of the 16 conditions, defined by left stimulus pair (same vs. different), right stimulus pair (same vs. different), and stimulus duration from stimulus onset to mask onset $(80,120,160$, or $280 \mathrm{msec})$. The order of trials within a block was randomized separately for each block.

Each trial began with the illumination of a fixation point. The subjects were instructed to press a foot switch when they fixated this point; they reported having no difficulty doing so-a report that has been corroborated in previous experiments with the same paradigm in which fixations were monitored. 8 The stimuli appeared $150 \mathrm{msec}$ after the foot switch was pressed. At the end of the stimulus duration selected for that trial, a mask was presented to terminate stimulus analysis, and it remained on the screen until the responses had been made. The mask consisted of two pairs of vertical lines that were all equal in length. They were superimposed over the stimulus lines, and they extended past the tops and bottoms of both lines in order to remove the length information provided by the stimulus.

Two versions of the experiment were run, in order, on all the subjects; the difference between versions was stimulus discriminability. In Experiment $1 \mathrm{~A}$, the outer line was $6.81^{\circ}$ long on different trials (high discriminability), and in Experiment $1 \mathrm{~B}$ it was $6.5^{\circ}$ long (medium discriminability).

The subjects responded at their leisure after the mask appeared; the instructions emphasized accuracy rather than latency. They first had to indicate "same" or "different" for each pair of stimulus lines, and then they had to indicate their confidence in each decision.

\section{Results and Discussion}

Following Bonnel et al. (1987), responses from the two sides were summarized by using standard procedures for estimating sensitivity and bias parameters from receiver operating characteristic (ROC) curves. "Same" versus "different" responses and confidence judgments were combined in order to obtain six response categories, from high-confidence same to high-confidence different. The five boundaries between adjacent pairs of these categories yielded five points on an ROC curve (see Macmillan \& Creelman, 1991, chap. 3), from which maximum likelihood parameter estimates were obtained with the procedures of Dorfman and Alf (1969). In order to increase the stability of the parameter estimates for the group data, responses were pooled across subjects before using these procedures (cf. Macmillan \& Kaplan, 1985). In additional analyses, we used the same procedures with unpooled (i.e., individual subjects) data.

Analyses of discrimination accuracy. Figure 3 shows discrimination accuracy as a function of attentional allocation and stimulus duration. Accuracy was measured by using the value of $d^{\prime}$ at the minor diagonal of the ROC curve $\left(d_{e}^{\prime}\right)$, because the estimated slopes of the binormal ROC curves were slightly different from one (cf. Egan \& Clarke, 1966). These values of $d^{\prime}$ were obtained by pooling responses across sides that received the same amount of attention (e.g., left responses from the $80 \% / 20 \%$ condition and right responses from the $20 \% / 80 \%$ condition), and very similar values were obtained by computing $d$ 's separately for each side and then averaging across sides.

Not surprisingly, the results demonstrate quite clearly that sensitivity increases with the proportion of attention devoted to the side and with the duration of the stimulus. The form of this increase is quantitatively very consistent with the sample-size model (Bonnel et al., 1987), which predicts that $d^{\prime 2}$ should increase linearly with the proportion of attention allocated to the side. The model substantially overpredicts $d^{\prime 2}$ for the conditions with the best performance ( 280 -msec duration and $80 \%$ or $100 \%$ of attention), but this may be due to something resembling a ceiling effect. Subjects may occasionally give wrong answers for reasons extraneous to their perceptual processing (e.g., accidentally pressing the wrong response key by sneezing during the trial), which would place an upper limit on the estimate of perceptual sensitivity, $d^{\prime 2}$.

Figure 4 shows performance separately for judgments about the left and right pairs of lines, using the format of a performance or attention operating characteristic (Sperling \& Melchner, 1978a). There is clear evidence of a tradeoff in task performance due to the allocation of attention, thus extending the findings of Bonnel et al. (1987) to tasks with masked stimuli. Intriguingly, there also appears to be an influence of stimulus duration on the form of the tradeoff (linear vs. curvilinear, in $d^{\prime}$ coordinates). This may also reflect a sort of ceiling effect on performance, however, if the highest $d^{\prime}$ estimates are suppressed by occasional unrepresentative trials.

As noted earlier, three of the models being compared - the sharing model and the deterministic singleand multiple-switch models-make relatively specific predictions about the functions relating $d^{\prime}$ to stimulus duration. We compared the accuracy of these predictions by fitting each of the three models to the observed $d$ 's. Fits were carried out separately for each individual subject, because pooling might artifactually worsen model 


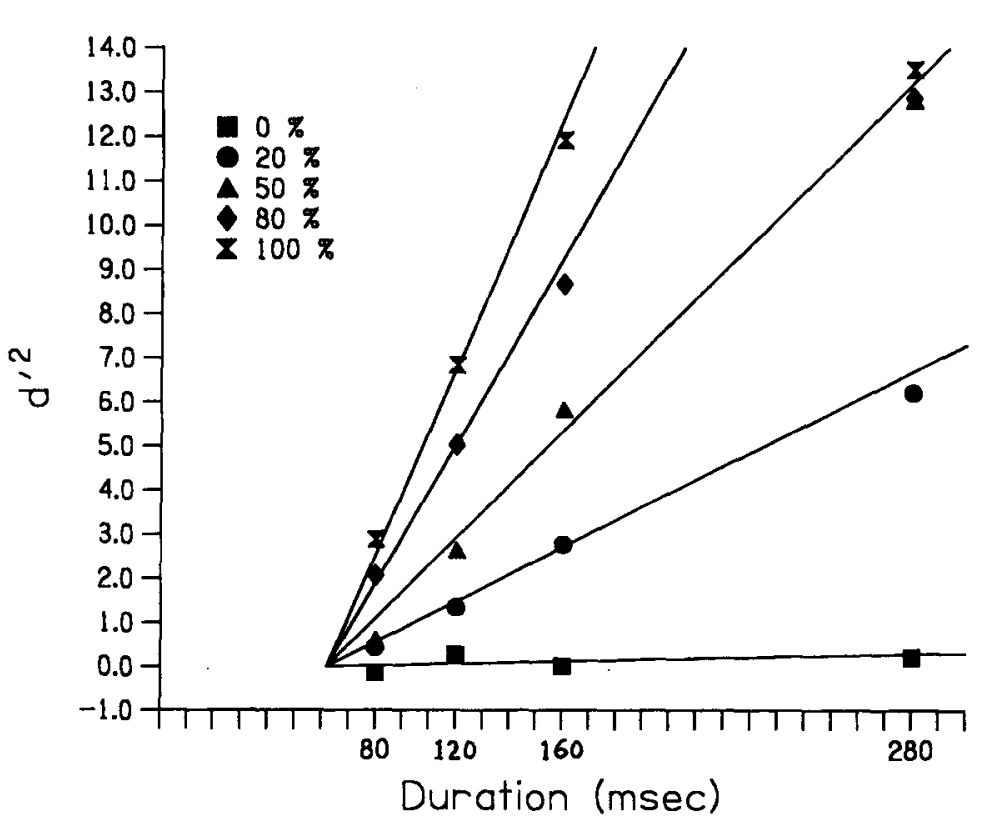

A

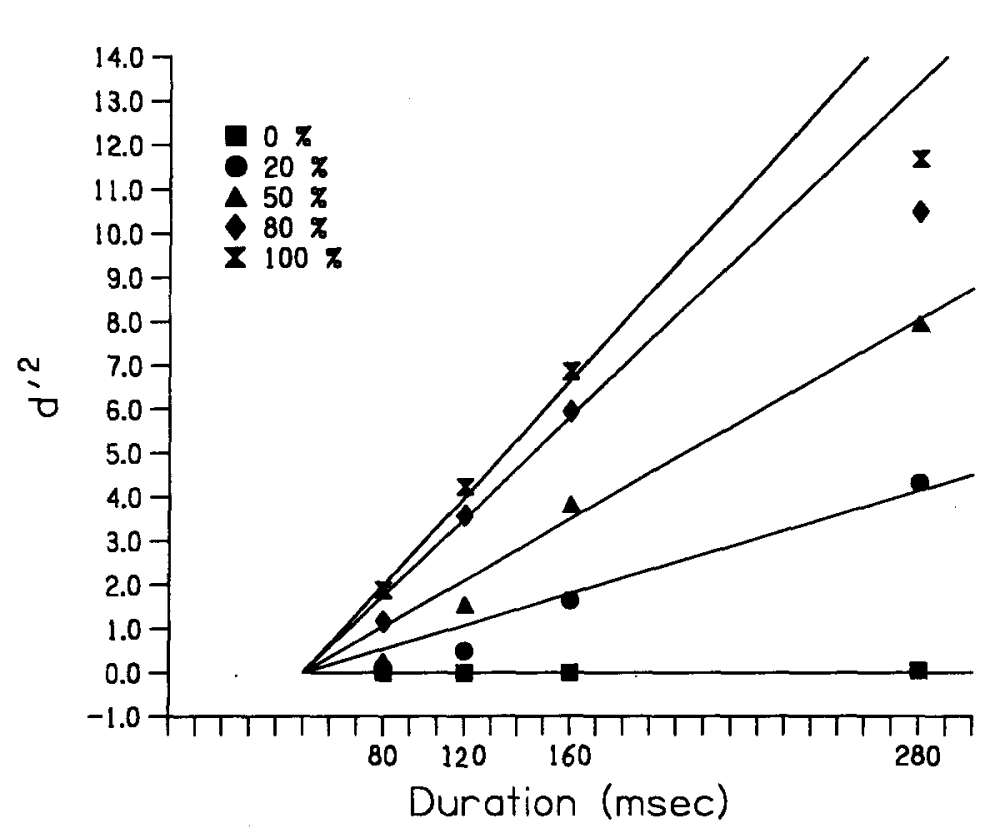

Figure 3. Experiments 1A and 1B: Sensitivity $\left(d^{\prime 2}\right)$ as a function of percentage of attention devoted to each side and stimulus duration $(80-280 \mathrm{msec})$. Observed values are shown as symbols, and the solid lines show the best-fitting straight lines, constrained to intersect at a common point, for each stimulus duration. The conditions with $80 \%$ and $100 \%$ of attention were excluded before fitting straight lines with stimulus durations of $280 \mathrm{msec}$, because the data suggest that a ceiling effect limited performance in those conditions. Panels $A$ and $B$ display results for Experiments $1 \mathrm{~A}$ and 1B, respectively. 

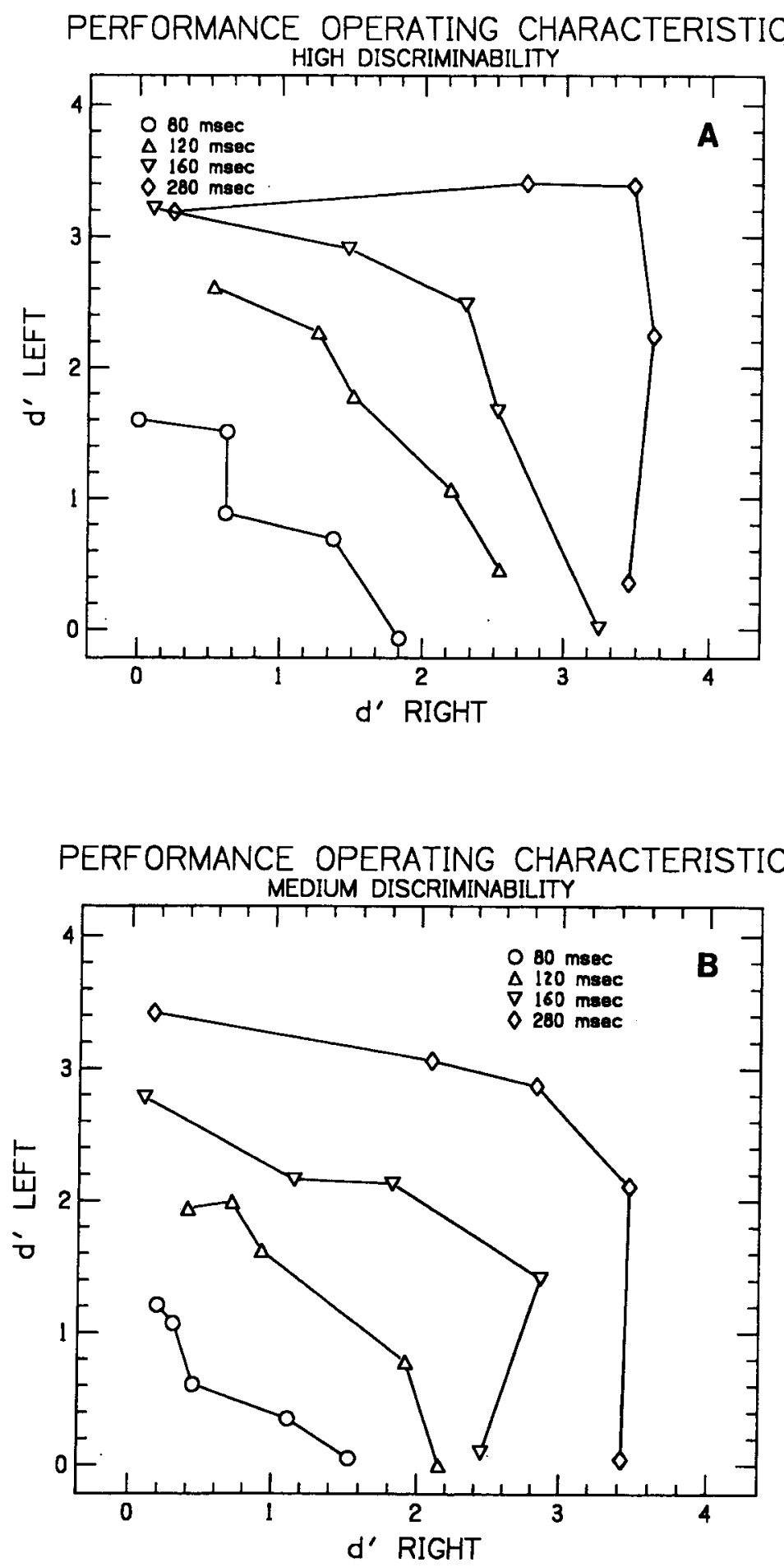

Figure 4. Experiments 1A and 1B: Performance operating characteristics showing joint performance on left and right discriminations as a function of stimulus duration. The different points on each curve were generated by varying attentional emphasis on the two sides. Panels $A$ and $B$ display results for Experiments $1 A$ and 1B, respectively. 
fits if different subjects adopted slightly different strategies, especially for the deterministic models (e.g., even if each subject performed in accordance with the deterministic single-switch model, pooled data could deviate from the model if different subjects chose different starting sides). The data to be predicted were the $40 d^{\prime}$ values observed for each subject (left vs. right side $\times 80$-, 120-, $160-$, or $280-\mathrm{msec}$ stimulus duration $\times 0 / 100,20 / 80$, $50 / 50,80 / 20$, or $100 / 0$ attentional allocation).

The sharing model we fit was simply the sample-size model, according to which $d^{\prime}$ is related to attentional condition $(a)$, side $(s)$, and stimulus duration $(D)$ by the function

$$
d^{\prime}(a, s, D)=\sqrt{\left(D-t_{0}\right) \times p(a, s) \times g_{a}} .
$$

The parameter $g_{a}$ indexes the rate at which perceptual information $\left(d^{\prime} 2\right)$ grows with the duration of the stimulus in the condition with full attention. The parameter $p(a, s)$ indicates the proportion of attention allocated to side $s$ in attentional condition $a$. These values are constrained to sum to 1.0 across sides within a given attentional condition, and they should be close but not necessarily identical to the instructed proportions of $0, .2$, $.5, .8$, and 1 . The parameter $t_{0}$ allows for the possibility that the subject cannot extract information from the stimulus during the entire time it is physically displayed, due to a start-up delay in processing (cf. Shibuya \& Bundesen, 1988; Townsend, 1981), and the need for such a parameter is strongly suggested by the fact that the functions displayed in Figures $3 \mathrm{~A}$ and $3 \mathrm{~B}$ intersect at stimulus durations greater than zero. Thus, this model has seven free parameters: $t_{0}, g_{a}, p(0 / 100$, left $), p(20 / 80$, left $)$, $p(50 / 50$, left $), p(80 / 20$, lef $t)$, and $p(100 / 0$, left $)$.

According to the deterministic single- and multipleswitch models,

$$
d^{\prime}(a, s, D)=\sqrt{d_{a}(a, s, D) \times g_{a}+d_{u}(a, s, D) \times g_{u}} .
$$

The free parameters $g_{a}$ and $g_{u}$ determine the rate at which perceptual information $\left(d^{\prime 2}\right)$ grows with the duration of attended and unattended processing, respectively. The durations of attended and unattended processing, $d_{a}(a, s, D)$ and $d_{u}(a, s, D)$, are determined in part by the stimulus duration $(D)$ and in part by nine other free parameters of the model:

1. A start-up delay, $t_{0}$, such that the subject does not begin accumulating perceptual information from the stimulus until $t_{0} \mathrm{msec}$ after its onset.

2 . The amount of time, $d_{a}(a)$, that the subject processes before switching sides in attentional condition $a$ $(0 \%, 20 \%, 50 \%, 80 \%$, or $100 \%)$.

3 . Whether the subject started by processing the left or right side in condition $a$. The starting side was a free parameter for the $20 / 80,50 / 50$, and $80 / 20$ attentional conditions, but it was assumed that the subject started on the $100 \%$ side in the $0 / 100$ and $100 / 0$ conditions.

Thus, both deterministic models had 11 free parameters - 4 more than the sample-size model. The two de- terministic models differed in how the durations of attended and unattended processing were computed, because the single-switch model allowed only one switch to take place, and the multiple-switch model allowed oscillations back and forth, if the stimulus duration was long enough relative to the length of the attentional moment $\left(d_{a}\right)$. Since each model is deterministic, though, it was a simple matter to compute the attended processing time, $d_{a}(a, s, d)$, for each side, given a starting side and switching time. The unattended processing time for the same side in the same condition was simply $D-t_{0}-$ $d_{a}(a, s, d)$.

For all three models, best-fitting parameter estimates were obtained by a numerical search algorithm (Rosenbrock, 1960), minimizing

$$
\sum_{i=1}^{40} \frac{\left(d_{o i}^{\prime}-\mathrm{E}\left[d_{i}^{\prime}\right]\right)^{2}}{\operatorname{Var}\left(d_{i}^{\prime}\right)}
$$

where $d_{o i}^{\prime}$ is the observed value of $d^{\prime}$ in the $i$ th condition, and $\mathrm{E}\left[d_{i}^{\prime}\right]$ and $\operatorname{Var}\left(d_{i}^{\prime}\right)$ are the predicted mean and variance of $d^{\prime}$ in that same condition. ${ }^{9}$ If the correct model is used to generate predicted values, this sum should be distributed approximately as a chi-square random variable, with degrees of freedom that are equal to the number of data points (i.e., 40) minus the number of free parameters of the model.

Table 1 summarizes the results. For each subject, it shows the error score obtained for the best-fitting version of each model. At the .01 significance level, the critical chi-square value is $\mathbf{5 4 . 8}$ for the sample-size model and 49.6 for the other two, so all three models can be rejected for every subject. This is disappointing, but not surprising, since the models ignore effects that are counterbalanced across subjects (e.g., order of testing in different conditions). A comparison of the relative merits of the three models shows that the single-switch model clearly provided the worst fits, and the samplesize and multiple-switch models provided fits that were

Table 1

Summary Error Measures Obtained in Fitting Sample-Size Model, Deterministic Single-Switch Model, and Deterministic Multiple-Switch Model to $d^{\prime}$ Values Obtained From Individual Subjects in Experiments $1 A$ and $1 B$

\begin{tabular}{cccc}
\hline & \multicolumn{3}{c}{ Model } \\
\cline { 2 - 4 } Subject & Sample-Size & Single-Switch & Multiple-Switch \\
\hline & & Experiment $1 \mathrm{~A}$ & \\
1 & 129.8 & 201.5 & 166.0 \\
2 & 101.9 & 118.8 & 108.8 \\
3 & 151.1 & 193.9 & 108.8 \\
4 & 310.1 & 317.3 & 247.1 \\
& & Experiment $1 \mathrm{~B}$ & \\
1 & 87.9 & 124.4 & 66.4 \\
2 & 181.7 & 240.2 & 63.9 \\
3 & 68.3 & 139.2 & 80.8 \\
4 & 81.3 & 179.9 & 127.1 \\
$M$ & 139.0 & 189.4 & \\
\hline
\end{tabular}


approximately equally good, despite the fact that the latter model has more than half again as many parameters as the former. Parameter estimates were reasonable for both of the latter two models. For the sample-size model, estimates of $t_{0}$ ranged from 0 to $49 \mathrm{msec}$ for different subjects, and estimates of $g_{a}$ ranged from .076 to .146 . For every subject, the estimated proportion of attention assigned to a side varied monotonically with the instructed proportion of attention to that side, and the estimated proportions were essentially $0 \%$ and $100 \%$ for the conditions with these instructed proportions. Similar estimates of $t_{0}$ and $g_{a}$ were obtained for the deterministic multiple-switch model. For the conditions with $20 \%, 50 \%$, and $80 \%$ of attention, in which switching would be expected to occur, the 24 estimated switching times ranged from 23 to $102 \mathrm{msec}$; the modal estimate was in the range of 50-60 msec and had a mean of $60 \mathrm{msec}$. Thus, the deterministic multiple-switch model generally does require multiple switches per trial to account for the accuracy data - a conclusion that is also apparent from the inadequate fits provided by the singleswitch model.

In sum, the discrimination accuracy data rule out the deterministic single-switch model and provide no reason for a preference of the deterministic multiple-switch model over the sharing model. Given the a priori implausibility of the deterministic multiple-switch model and its failure to provide a fit that was noticeably better than that for the sample-size model, in spite of its larger number of parameters, we conclude that the sharing model provides a better account of the accuracy data than either of these two competitors. Having rejected two types of switching models, we turn now to an examination of the contingency data, in order to see whether contingencies were negative, as predicted by stochastic switching models, or zero, as predicted by sharing models.

Contingency analyses. "Same" and "different" responses were scored as correct or incorrect. Each trial was tabulated in a $2 \times 2$ table; rows indicated the accuracy of one task and columns indicated the accuracy of the other task. Separate tables were constructed for each combination of attentional condition and stimulus duration. The phi coefficient was used to measure the contingency observed in each $2 \times 2$ table (Hays, 1973, Sec. 17.12). Table 2 shows the observed phi coefficient for each combination of stimulus duration and attentional allocation. Each phi is based on 288 observations pooled across 4 subjects, and not one is sufficiently negative to reject the null hypothesis of zero contingency $(p<.05)$. On the other hand, a number of positive phi coefficients (i.e., those exceeding .12) are significantly different from zero. Unfortunately, it is difficult to interpret positive phi coefficients computed by pooling across subjects, because such pooling would produce positive contingencies if subjects differed in overall sensitivity.

To avoid pooling problems, these same phi coefficients were also computed individually for each of the
Table 2

Experiments 1A and 1B: Observed Phi Coefficients as

a Function of Stimulus Duration (in Milliseconds) and Attentional Condition

\begin{tabular}{|c|c|c|c|c|}
\hline \multirow{2}{*}{$\begin{array}{l}\text { Attention } \\
\text { Condition } \\
\text { (Percent) }\end{array}$} & \multicolumn{4}{|c|}{ Stimulus Duration } \\
\hline & 80 & 120 & 160 & 280 \\
\hline \multicolumn{5}{|c|}{ Experiment $1 \mathrm{~A}$ : High Discriminability } \\
\hline $20 / 80$ & 0.03 & -0.03 & 0.21 & 0.22 \\
\hline $50 / 50$ & 0.06 & 0.18 & 0.34 & 0.36 \\
\hline $80 / 20$ & -0.04 & -0.02 & 0.12 & 0.26 \\
\hline \multicolumn{5}{|c|}{ Experiment 1B: Medium Discriminability } \\
\hline $20 / 80$ & 0.11 & 0.01 & 0.14 & -0.06 \\
\hline $50 / 50$ & 0.04 & 0.14 & 0.02 & 0.02 \\
\hline $80 / 20$ & -0.10 & 0.10 & 0.03 & -0.05 \\
\hline
\end{tabular}

Note-Phi coefficients were computed from 288 observations per condition, pooled across 4 subjects. The critical phi values are \pm 0.115 and \pm 0.152 for $p<.05$ and $p<.01$, respectively.

subjects $(N=72$ observations). Of the 160 individualsubject phi's thus computed (Experiment $1 \mathrm{~A}$ vs. $1 \mathrm{~B} \times 4$ subjects $\times 4$ stimulus durations $\times 5$ attention conditions), only one was sufficiently negative to reject the null hypothesis of zero contingency $(p<.05$, two-tailed, in the condition with a duration of $280 \mathrm{msec}$ and $100 \%$ of attention paid to the left side). On the other hand, 16 of the 160 contingencies computed for individual subjects were significant in the positive direction. ${ }^{10}$

Overall, the contingency analyses indicate that a correct response on one side definitely does not predict an error on the other. This pattern is inconsistent with the negative contingencies predicted by the stochastic switching models, and instead conforms to the approximate independence predicted by sharing models. ${ }^{11}$

Because only 1 of 160 contingencies was significant in the negative direction, and 16 of 160 were significant in the positive direction, it is tempting to conclude that there are actually positive within-condition contingencies. Such positive contingencies could be caused by trial-to-trial fluctuations that subtly influence performance on both tasks in the same way (e.g., alertness, practice level, etc.), or by the existence of occasional trials on which subjects give wrong answers for reasons extraneous to their perceptual processing (e.g., blinks). Alternatively, however, this pattern may be a statistical artifact. It is well known that the chi-square statistic computed from $2 \times 2$ tables only approximately follows the chi-square distribution (e.g., Marascuilo, 1971), and simulations 12 of a model in which independence held revealed that Type I errors are much more likely to occur with positive than with negative contingencies, especially when the probability of a correct response exceeds .8. Thus, the larger number of significant positive than negative contingencies, especially in conditions with high percentages of correct responses, could be due to differing Type I error probabilities.

Analyses of response latencies. Mean response latency was $809 \mathrm{msec}$ on single-response trials (attention conditions $0 / 100$ and 100/0), and latencies were 1,307 , 
1,371 , and 1,612 msec on double-response trials with $80 \%, 50 \%$, and $20 \%$ of attention, respectively, so the subjects tended to respond first to the side with the higher priority. On average, latency decreased from 1,669 to $892 \mathrm{msec}$ as stimulus duration increased from 80 to $280 \mathrm{msec}$, in keeping with the usual finding that easier tasks yield faster responses.

Conclusions. The lack of negative within-task contingencies favors sharing over switching explanations of attention division in dual-task line-length discriminations. Switching models can only be reconciled with the absence of such contingencies by assuming that switching is either very fast or deterministic. However, even the deterministic switching models require many fast switches within a single trial in order to account for the accuracy results. Thus, the only switching models that seem compatible with the present results are those that allow very fast switching - at least every $50 \mathrm{msec}$-and, as argued in the introduction, such models are very implausible.

\section{EXPERIMENT 2}

The results of Experiment 1 provide evidence against switching models with switches occurring, at most, once every $50 \mathrm{msec}$, and we have argued in the introduction that models with even faster switching are implausible in the first place. Nonetheless, it would be more decisive to have further evidence against fast switching models from within this paradigm. The purpose of Experiment 2 was to provide such evidence by using very brief $(20 \mathrm{msec})$ stimulus presentations. Masks were presented at stimulus offset in order to terminate perceptual analysis.

One reason for using brief presentations is that it forces switching models to predict negative contingencies even when they have relatively fast switching rates. Suppose, for example, that switches could occur after $20 \mathrm{msec}$ of processing-very fast switching indeed. Nonetheless, on each trial of this experiment, the subject could attend to only one side, because the brief presentation would end when it was time to switch. In essence, brief presentations force the subject into a trial-by-trial switching strategy unless switching is extremely rapid (i.e., more often than once per $20 \mathrm{msec}$ ). And, as we have already seen, trial-by-trial switching predicts strong negative contingencies (cf. Sperling \& Melchner, 1978a, 1978b).

A second prediction of switching models for this experiment is based on an inherent limitation on switching performance for dual-task situations (cf. Baron, 1973; Shiffrin, Pisoni, \& Castaneda-Mendez, 1974, Equation 1; Veniar, 1958). Suppose that, on each trial, a subject attends to one task or the other-but never bothand that the subject obtains information only about the attended task. In this case, the subject can achieve a maximum performance level of $75 \%$ correct, pooling across trials and tasks. This is because the subject can do no better than to respond perfectly on each trial's at- tended task ( $100 \%$ correct) and to guess on each unattended one $(50 \%)$.

If subjects have an attentional mechanism that switches less often than once every $20 \mathrm{msec}$, then on each trial they should be able to attend to only one task or the other, and they should get no more than $75 \%$ correct. On the other hand, if they can truly share attention-or switch more often than once every $20 \mathrm{msec}$, which we regard as highly unlikely for reasons discussed previouslythen they may well be able to perform better than $75 \%$ correct overall by obtaining some information about both stimulus pairs presented on a single trial. Of course, if the discrimination is sufficiently difficult, especially given the brief stimulus duration, they might not exceed $75 \%$ correct, even when attention is shared. To maximize the chances that subjects would exceed $75 \%$ correct if they were indeed sharing, we used line lengths that corresponded to the high-discriminability condition of Experiment 1.

An assumption that is critical to this prediction is that subjects extract no information from an unattended stimulus pair. If they do obtain some information without attention, then they might perform better than chance on the unattended task, and hence perform better than $75 \%$ correct overall. Consistent with this assumption is the fact that previous experiments with this paradigm have yielded performance at or very near chance when subjects were instructed to pay $0 \%$ of their attention to a particular pair of lines (e.g., Bonnel \& Miller, 1994; see also the present Experiment 1). The only difference between the present experiment and these previous ones is the major reduction in stimulus duration, and it is difficult to see how this change could obviate the need for attention.

One important precaution that we took was to vary the moment of stimulus onset unpredictably, so that the subjects would not know in advance exactly when the stimuli would appear. If this were not done, it would be possible for them to obtain some information about each stimulus pair, even with an attentional mechanism that switched infrequently (e.g., no more often than once per $100 \mathrm{msec}$ ). With a predictable stimulus onset, the danger is that the subjects could plan in advance to switch attention just after stimulus onset (e.g., exactly $10 \mathrm{msec}$ after the stimulus appeared). If they could make such well-timed attentional switches, they could, in principle, attend to both stimulus pairs on a given trial, even though the attentional mechanism could not switch very often. This strategy is implausible a priori, because it requires extremely accurate temporal anticipation and a high degree of precision in the moment of switching attention. Nonetheless, it was easy enough to eliminate the strategy altogether by using an unpredictable moment of stimulus onset.

Another important precaution was that we used very effective masks, which ensured that no information could be obtained from a slow switch (i.e., more than $20 \mathrm{msec}$ after stimulus onset). We used the same masks that we had used in Experiment 1 (i.e., long lines super- 
imposed over the stimulus lines). Regardless of whether these masks interrupt stimulus processing or are merely integrated with the stimulus representations, they should abruptly end the uptake of new information about the lengths of the original stimuli because, by construction, they remove all stimulus features on which the discrimination might be based (cf. Eriksen, 1980).

\section{Method}

Except as described below, the apparatus and procedure were identical to those of Experiment 1.

The subjects were 6 employees of the C.N.R.S., none of whom had served in Experiment 1. Each subject was tested in 16 blocks of 96 trials, equally divided across four sessions. Four blocks were devoted to each of four attention conditions $(20 \% / 80 \%, 50 \% / 50 \%$, $80 \% / 20 \%$, and single-task). The order of conditions was balanced across subjects and sessions. Within each block, trials were equally divided among the four combinations of same versus different left stimulus pair, and same versus different right stimulus pair.

After the subject pressed the foot switch to begin the trial, there was a random delay of $150+E \mathrm{msec}$, where $E$ was an exponentially distributed random variable with a mean of $1 \mathrm{sec}$. Then the stimulus display appeared for $20 \mathrm{msec}$, after which the mask appeared and the subject responded.

\section{Results and Discussion}

As in Experiment 1, contingencies were computed for each subject under each of the divided-attention conditions $(20 \% / 80 \%, 50 \% / 50 \%$, and $80 \% / 20 \%)$. Of these 18 contingencies, 9 were negative, 1 was significantly so $(p<.05)$, and 2 approached significance $(p<.10)$. On the other hand, 3 were significantly positive $(p<.01)$, and another approached significance $(p<.10)$. Overall, the absence of strong, negative within-condition contingencies indicates that the tasks do not trade off against one another from trial to trial, as would be expected if subjects obtained information about only one task per trial. This finding is strong evidence against models in which switching occurs less often than approximately once per $20 \mathrm{msec}$.

Further evidence against such switching models comes from the percentages of correct responses (PCs), which are shown for each subject and condition in Table 3. Averaging across subjects and sides, single-task PC was $92.0 \%$, and PCs were $85.8 \%, 84.5 \%$, and $85.3 \%$ in conditions with $20 \%, 50 \%$, and $80 \%$ of attention, respectively. The difference between single-task performance and the average of the dual-task conditions was significant $[t(5)=2.84, p<.05]$, as was the difference between single-task performance and performance with $50 \%$ of attention $[t(5)=3.83, p<.02]$. Single-task performance was not reliably better than performance with $20 \%[t(5)=1.80, p>.10]$ or $80 \%[t(5)=2.04,0.10>$ $p>.09]$ of attention, and there were no differences in overall performance in the conditions with $20 \%, 50 \%$, and $80 \%$ of attention (all $p \mathrm{~s}>.50$ ).

The PCs clearly rule out models in which information is obtained about only one task per trial. Mean accuracy was significantly above $75 \%$ in all three attention conditions $[50 \% / 50 \%, t(5)=5.31, p<.005 ; 20 \% / 80 \%, t(5)=$
Table 3

Experiment 2: Percentage Correct as a Function of Condition

\begin{tabular}{|c|c|c|c|c|c|c|c|c|}
\hline \multirow[b]{2}{*}{ Subject } & \multicolumn{2}{|c|}{ Single-Task } & \multicolumn{2}{|c|}{$50 \% / 50 \%$} & \multicolumn{2}{|c|}{$20 \% / 80 \%$} & \multicolumn{2}{|c|}{$80 \% / 20 \%$} \\
\hline & Left & Right & Left & Right & Left & Right & Left & Right \\
\hline 1 & 94 & 92 & 89 & 71 & 84 & 88 & 86 & 79 \\
\hline 2 & 93 & 80 & 87 & 83 & 90 & 84 & 89 & 88 \\
\hline 3 & 98 & 96 & 93 & 88 & 81 & 76 & 73 & 73 \\
\hline 4 & 92 & 88 & 83 & 76 & 91 & 82 & 88 & 86 \\
\hline 5 & 89 & 93 & 92 & 85 & 93 & 87 & 87 & 90 \\
\hline 6 & 95 & 93 & 87 & 80 & 87 & 91 & 93 & 88 \\
\hline$M$ & 93.5 & 90.3 & 88.5 & 80.5 & 87.7 & 84.7 & 86.0 & 84.0 \\
\hline
\end{tabular}

$6.74, p<.005 ; 80 \% / 20 \%, t(5)=3.79, p<.02]$, which is the maximum that any such model can predict. ${ }^{13}$

Rather surprisingly, there was no effect of the instructed proportion of attention (i.e., $20 \%, 50 \%$, or $80 \%$ ) on accuracy. Strong effects of attention on discrimination accuracy have consistently been found in previous studies with this paradigm (e.g., Bonnel et al., 1987), although a similar null effect of attention has been found in dual-task studies of the detection of luminance changes (Bonnel, Stein, \& Bertucci, 1992). The present study differed from earlier ones mainly in the use of much briefer stimulus presentations ( $20 \mathrm{vs}$. approximately $100 \mathrm{msec}$ ); further research will be needed to determine why such brief presentations eliminated the effect of attention.

The lack of an attention effect appears to cast doubt on the conclusion that subjects attended simultaneously to both tasks in order to surpass $75 \%$ correct. An alternative explanation is that attention was not needed-or even useful-for performing the task under the present conditions, and that responses were based solely on unattended processing. If that were the case, of course, the fact that information is obtained from both tasks on a single trial would not result from both tasks' being attended on a single trial. This alternative explanation seems extremely implausible, however, because of previous evidence that line-length discriminations require attention with longer stimulus durations (e.g., Bonnel \& Miller, 1994; Bonnel et al., 1987; see also the present Experiment 1). Another possibility is that some attentional control operations take place after stimulus onset, and that the brief stimulus durations used here did not allow sufficient time for them. If such control operations were required for the fine tuning of attentional allocation, there might not have been enough time in this experiment to set up differential processing for tasks assigned $20 \%, 50 \%$, or $80 \%$ of attention.

One rather surprising finding is that overall performance was actually slightly worse with the 80 -msec displays in Experiment $1 \mathrm{~A}$ than with the 20 -msec displays of the same stimuli in Experiment 2. Across all conditions, mean accuracy was $74 \%$ correct with the $80-\mathrm{msec}$ displays in Experiment 1A, compared with an average of $87 \%$ in Experiment $2[t(8)=9.0, p<.001]$. The difference was primarily due to the fact that accuracy did not decrease as attention decreased in Experiment 2. The differences between the two experiments in percentages 
correct were $5 \%, 7 \%, 18 \%$, and $22 \%$ for conditions with $100 \%, 80 \%, 50 \%$, and $20 \%$ of attention, respectively. The latter two differences were highly reliable statistically $(p<.001)$, whereas the former two were not statistically reliable $(p>.05)$. In addition to being less accurate in the former experiment, responses were also somewhat slower.

There are a number of possible procedural differences that could account for the subjects' superior performance in Experiment 2. The most obvious difference is that display duration was varied randomly in the former experiment, but was fixed in the latter. Any type of uncertainty about the signal adds noise to the decision process (cf. Green \& Swets, 1966), and the superiority shown in Experiment 2 could have resulted from the absence of such noise. A second procedural difference concerns the subjects: those in Experiment 1 had had previous experience in a version of the task with unmasked stimulus displays, whereas those in Experiment 2 had not had previous experience with the task. It is possible that the masks caused more interference for the former subjects, because they had previously developed strategies appropriate to maskless displays and were unable to change to new strategies.

Another possible explanation for the superior performance shown with shorter displays is simply that performance may be a U-shaped function of SOA under these conditions. Although it is somewhat counterintuitive that performance should first decrease and then increase with increases in the time between stimulus and mask, such nonmonotonic functions are in fact the rule in metacontrast situations (e.g., Bernstein, Proctor, Belcher, \& Schurman, 1974). Moreover, U-shaped functions have also been observed in discrimination tasks when the masks are superimposed over the target stimuli-that is, under conditions fairly similar to those of the present experiments (e.g., Michaels \& Turvey, 1973; Purcell \& Stewart, 1970; Purcell, Stewart, \& Hochberg, 1982; Schwartz \& Pritchard, 1981; Stewart \& Purcell, 1974; Turvey, 1973, Experiment 18). In one condition of Turvey's Experiment 18, for example, discrimination was clearly better with an SOA of $16 \mathrm{msec}$ than with an SOA of $80 \mathrm{msec}$. The reasons for such Ushaped masking functions are not yet entirely clear, but they may be due to differing time courses of peripheraland central-masking processes (Turvey, 1973) or of processes that are sensitive to high and low spatial frequency (Breitmeyer, 1975). In any case, such a function may be responsible for the fact that performance was better in Experiment 2 than that with the 80 -msec displays of Experiment $1 \mathrm{~A}$.

\section{GENERAL DISCUSSION}

Overall, the present results support the view that subjects can attend simultaneously to two demanding perceptual discriminations rather than switching back and forth between them, either across trials or within a trial. The main finding of Experiment 1 - that an accurate re- sponse on one discrimination task is not associated with an inaccurate response on the other task, even when stimulus duration is unpredictable - is inconsistent with trial-to-trial switching models that have been supported in other tasks (e.g., Sperling \& Melchner, 1978b) and is also inconsistent with most within-trial switching models in which switches occur less often than approximately every $50 \mathrm{msec}$. Although it is possible to model these data by assuming that switching occurs more than 20 times per second, the assumption of very rapid switching is not plausible within a neurophysiological architecture that seems much more suited to slow parallel computations than to fast serial ones (e.g., Kolb \& Whishaw, 1990). Thus, the most natural explanation for the lack of negative contingency is that the subjects simply divided their attention between the two tasks in approximately the same proportion on each trial, holding this allocation constant for the entire stimulus exposure. According to this "sharing" view of attention, accuracy on one task is uncorrelated with accuracy on the other task because the tasks do not trade resources back and forth, either across trials or within a trial.

The results of Experiment 2 provided further evidence of sharing with $20-\mathrm{msec}$, masked stimulus displays. Negative contingencies were still absent with these brief displays, and switching models would require very high switching rates to accommodate their absence. Furthermore, the accuracy data indicated that the subjects obtained some information about both tasks within a single 20-msec stimulus display. This finding is also quite compatible with the view that subjects can attend to two tasks simultaneously, extracting a little information concerning each discrimination during the brief display. A switching model would have to oscillate very rapidly to get information from both locations within a single 20-msec presentation (more than 50 switches per second!), and there is no reason to believe that such rapid switching is within the capability of the human attentional system. The lack of an attention effect $(20 \%$ vs. $50 \%$ vs. 80\%) in Experiment 2 leaves open the possibility that information was obtained with unattended processing, but previous evidence of the importance of attention in making these discriminations makes this interpretation very unlikely.

The present experiments extend previous examinations of switching versus sharing models to a new task, and it is reasonable to consider whether any general conclusion about the two models can yet be reached. In brief, evidence of switching has been found only when subjects must monitor multiple locations in order to detect a target letter (Shaw, 1982; Sperling \& Melchner, 1978a, 1978b). Evidence of sharing has been found when subjects must monitor multiple locations to determine stimulus onset times (Baron, 1973) or brightness changes (Hughes, 1984; Pylyshyn \& Storm, 1988), or when they need to make line-length comparisons (current experiments). This pattern suggests the intriguing hypothesis that attention is switched only when the task requires information about conjunctions of features (cf. 
Treisman, 1986), because the letter-detection task is the only one that seems to require conjunction processing. On the other hand, Townsend (1981) also found evidence of attentional sharing (i.e., independent detections) in a whole-report task, for which attention must be divided across all of the letters in a given display. It seems likely that conjunction processing was also required in this task, in which case the observed independence would contradict the hypothesis just advanced. However, Townsend used stimulus letters from a typewriter font, leaving open the possibility that the letters were detected by recognizing unique features rather than by recognizing conjunctions.

In conclusion, we note that the question of sharing versus switching is also of interest with respect to the general division between capacity theories and spotlight theories of spatial attention (e.g., Shaw, 1978; Umiltà, 1988). The present evidence of sharing is certainly compatible with capacity models (cf. Shaw, 1980, 1982; Sperling, 1984; Sperling \& Melchner, 1978a, 1978b), because these models permit arbitrary assignment of attentional resources across locations. It is also compatible with spotlight models that allow multiple locations to receive attention simultaneously (e.g., models including a diffuse attentional state or a gradient of attention), but it is incompatible with more restrictive spotlight models that allow only one attended location per trial (cf. Umiltà, 1988).

\section{REFERENCES}

Baron, J. (1973). Division of attention in successiveness discrimination. In S. Kornblum (Ed.), Attention and performance IV (pp. 703711). New York: Academic Press.

Bernstein, I. H., Proctor, R. W., Belcher, J., \& Schurman, D. L. (1974). An analysis of U-shaped metacontrast. Perception \& Psychophysics, 16, 329-336.

BONNEl, A.-M., \& MiLleR, J. (1994). Attentional effects on concurrent psychophysical discriminations: Investigations of a sample-size model. Perception \& Psychophysics, 55, 162-179.

BonNel, A.-M., Possamai, C. A., \& Schmitt, M. (1987). Early modulation of visual input: A study of attentional strategies. Quarterly Journal of Experimental Psychology, 39A, 757-776.

Bonnel, A.-M., Stein, J. F., \& BertuCCI, P. (1992). Does attention modulate the perception of luminance changes? Quarterly Journal of Experimental Psychology, 44A, 601-626.

Breitmeyer, B. G. (1975). Predictions of ' $U$ '-shaped backward pattern masking from considerations of the spatio-temporal frequency response. Perception, 4, 297-304.

CRICK, F. H. C., \& Asanuma, C. (1986). Certain aspects of the anatomy and physiology of the cerebral cortex. In J. L. McClelland \& D. E. Rumelhart (Eds.), Parallel distributed processing: Explorations in the microstructure of cognition: Vol. 2. Psychological and biological models (pp. 333-371). Cambridge, MA: MIT Press.

Dorfman, D. D., \& ALF, E., JR. (1969). Maximum-likelihood estimation of parameters of signal-detection theory and determination of confidence intervals: Rating method data. Journal of Mathematical Psychology, 6, 487-496.

Egan, J. P., \& Clarke, F. R. (1966). Psychophysics and signal detection. In J. B. Sidowsky (Ed.), Experimental methods and instrumentation in psychology (pp. 211-246). New York: McGraw-Hill.

ERIKSEN, C. W. (1980). The use of a visual mask may seriously confound your experiment. Perception \& Psychophysics, 28, 89-92.

Eriksen, C. W., Goettl, B., St. James, J. D., \& Fournier, L. R. (1989). Processing redundant signals: Coactivation, divided attention, or what? Perception \& Psychophysics, 45, 356-370.
ERIKSEN, C. W., \& MURPhY, T. D. (1987). Movement of attentional focus across the visual field: A critical look at the evidence. Perception \& Psychophysics, 42, 299-305.

ESTES, W. K., \& WESSEL, D. L. (1966). Reaction time in relation to display size and correctness of response in forced-choice visual signal detection. Perception \& Psychophysics, 1, 369-373.

Gourevitch, V., \& Galanter, E. (1967). A significance test for one parameter isosensitivity functions. Psychometrika, 32, 25-33.

Green, D. M., \& LuCE, R. D. (1974). Variability of magnitude estimates: A timing theory analysis. Perception \& Psychophysics, 15, 291-300.

GreEN, D. M., \& SWETS, J. A. (1966). Signal detection theory and psychophysics. New York: Wiley.

HaYs, W. (1973). Statistics for the social sciences. New York: Holt, Rinehart \& Winston.

Hughes, H. C. (1984). Effects of flash luminance and positional expectancies on visual response latency. Perception \& Psychophysics, 36, 177-184.

JAMES, W. (1890). Principles of psychology. New York: Holt.

JONIDES, J. (1980). Towards a model of the mind's eye's movement. Canadian Journal of Psychology, 34, 103-112.

KLEIN, R. M. (1988). Inhibitory tagging system facilitates visual search. Nature, 334, 430-431.

KolB, B., \& WHISHAW, I. Q. (1990). Fundamentals of human neuropsychology (3rd ed.). New York: W. H. Freeman.

LUCE, R. D. (1977). Thurstone's discriminal processes fifty years later. Psychometrika, 42, 461-489.

Macmillan, N. A., \& Creelman, C. D. (1991). Detection theory: A user's guide. Cambridge: Cambridge University Press.

Macmillan, N. A., \& Kaplan, H. L. (1985). Detection theory analysis of group data: Estimating sensitivity from average hit and falsealarm rates. Psychological Bulletin, 98, 185-199.

MARAsCuILO, L. A. (1971). Statistical methods for behavioral science research. New York: McGraw-Hill.

MARR, D. (1982). Vision. New York: W. H. Freeman.

MAYLOR, E. A., \& HOCKEY, R. J. (1985). Inhibitory component of externally controlled covert orienting in visual space. Journal of Experimental Psychology: Human Perception \& Performance, 11, 777-787.

Michaels, C. F., \& Turvey, M. T. (1973). Hemiretinae and nonmonotonic masking functions with overlapping stimuli. Bulletin of the Psychonomic Society, 2, 163-164.

NeISSER, U. (1963). Decision time without reaction time: Experiments in visual scanning. American Journal of Psychology, 76, 376-385.

PoSNER, M. I., \& CoHEN, Y. (1984). Components of visual orienting. In H. Bouma \& D. G. Bowhuis (Eds.), Attention and performance X: Control of language processes (pp. 531-556). Hillsdale, NJ: Erlbaum.

PURCELl, D. G., \& STEWART, A. L. (1970). U-shaped backward masking functions with nonmetacontrast paradigms. Psychonomic Science, 21, 361-363.

Purcell, D. G., STewart, A. L., \& Hochberg, E. P. (1982). Recovery and nonmonotone masking effects. Vision Research, 22, 10871091.

Pylyshyn, Z. W., \& Storm, R. W. (1988). Tracking multiple independent targets: Evidence for a parallel tracking mechanism. Spatial Vision, 3, 179-197.

Remington, R., \& PierCe, L. (1984). Moving attention: Evidence for time-invariant shifts of visual selective attention. Perception \& Psychophysics, 35, 393-399.

RoSENBROCK, H. H. (1960). An automatic method for finding the greatest or least value of a function. Computer Journal, 3, 175-184.

SCHNEIDER, W., \& SHIFFrIN, R. M. (1977). Controlled and automatic human information processing: I. Detection, search, and attention. Psychological Review, 84, 1-66.

Schwartz, M., \& Pritchard, W. S. (1981). AERs and detection in tasks yielding 'U'-shaped backward masking functions. Psychophysiology, 18, 678-685.

SCHWEICKERT, R., \& BogGs, G. J. (1984). Models of central capacity and concurrency. Journal of Mathematical Psychology, 28, 223-281.

SEJNOWSKI, T. J. (1986). Open questions about computation in cerebral cortex. In J. L. McClelland \& D. E. Rumelhart (Eds.), Parallel 
distributed processing: Explorations in the microstructure of cognition: Vol. 2. Psychological and biological models (pp. 372-389). Cambridge, MA: MIT Press.

SHAw, M. L. (1978). A capacity allocation model for reaction time. Journal of Experimental Psychology: Human Perception \& Performance, 4, 586-598.

SHAW, M. L. (1980). Identifying attentional and decision-making components in information processing. In R. S. Nickerson (Ed.), Attention and performance VIII (pp. 277-296). Hillsdale, NJ: Erlbaum.

SHAW, M. L. (1982). Attending to multiple sources of information: I. The integration of information in decision making. Cognitive Psychology, 14, 353-409.

ShibuYa, H., \& BUNDESEN, C. (1988). Visual selection from multielement displays: Measuring and modeling effects of exposure duration. Journal of Experimental Psychology: Human Perception \& Performance, 14, 591-600.

Shiffrin, R. M., Pisoni, D. B., \& Castaneda-Mendez, K. (1974). Is attention shared between the ears? Cognitive Psychology, 6, 190-215.

Shulman, G. L., Remington, R., \& MCLean, J. P. (1979). Moving attention through visual space. Journal of Experimental Psychology: Human Perception \& Performance, 5, 522-526.

SPERLING, G. (1984). A unified theory of attention and signal detection. In R. Parasuraman \& D. R. Davies (Eds.), Varieties of attention (pp. 103-182). New York: Academic Press.

SPERLING, G., \& MELCHNER, M. (1978a). The attention operating characteristic: Examples from visual search. Science, 202, 315-318.

SPERLING, G., \& Melchner, M. (1978b). Visual search, visual attention, and the attention operating characteristic. In J. Requin (Ed.), Attention and performance VII (pp. 675-686). Hillsdale, NJ: Erlbaum.

SPERLING, G., \& REAVES, A. (1980). Measuring the reaction time of a shift of visual attention. In R. S. Nickerson (Ed.), Attention and performance VIII (pp. 347-360). Hillsdale, NJ: Erlbaum.

Stewart, A. L., \& Purcell, D. G. (1974). Visual backward masking by a flash of light: A study of U-shaped detection functions. Journal of Experimental Psychology, 103, 553-566.

TOWNSEND, J. T. (1981). Some characteristics of visual whole report behavior. Acta Psychologica, 47, 149-173.

Treisman, A. M. (1986). Features and objects in visual processing. Scientific American, 254, 114-124.

Treisman, A. M., \& Gelade, G. (1980). A feature-integration theory of attention. Cognitive Psychology, 12, 97-136.

TSAL, Y. (1983). Movements of attention across the visual field. Journal of Experimental Psychology: Human Perception \& Performance, 9, 523-530.

Turvey, M. T. (1973). On peripheral and central processes in vision: Inferences from an information-processing analysis of masking with patterned stimuli. Psychological Review, 80, 1-52.

UMILTÀ, C. (1988). Orienting of attention. In F. Boller \& J. Grafman (Eds.), Handbook of neuropsychology (Vol. 1, pp. 175-193). Amsterdam: Elsevier.

VENIAR, F. A. (1958). Signal detection as a function of frequency ensemble: I. Journal of the Acoustical Society of America, 30, 10201024.

\section{NOTES}

1. For completeness, we note that, in this task, the trial-to-trial switching model is also contradicted by the observed function relating discrimination performance on one side to the attentional emphasis on that side. This model predicts that the percentage of correct responses should be linearly related to the proportion of attention (Sperling \& Melchner, 1978a, 1978b); instead, $d^{\prime 2}$ has been observed to be a linear relation of attention (e.g., Bonnel et al., 1987).

2. One might want to examine the literature on attentional movement and visual search more closely to see whether switching really could be fast enough to be useful in a dual-task situation. Unfortunately, how to estimate the maximum switching speed from data concerning attentional movement or visual search is not clear. Clearly, for example, visual search rate does not simply reflect the time to move attention, because it is also affected by the time needed to reject nontarget items (e.g., Neisser, 1963). Similar difficulties arise in trying to extract a maximum switching rate from studies of attentional movement. For example, Sperling and Reaves (1980) estimated 200-250 msec as the reaction time for an attentional shift, but this estimate includes the time needed to detect and interpret the visual signal commanding the shift, so it too is clearly an overestimate of the minimum time between attentional switches. Analogous problems arise in trying to estimate the speed of attentional movement from data obtained in other paradigms (see Eriksen \& Murphy, 1987).

3. To avoid confusion about terminology, we must emphasize that, in the present article, we do not use the terms switching and sharing in the same way that Shaw $(1980,1982)$ and Sperling and Melchner $(1978 \mathrm{a}, 1978 \mathrm{~b}) \mathrm{do}$. They used the term switching models to refer only to trial-to-trial switching, including within-trial switching models in the "sharing" class. Shaw (1980), for example, defined sharing models: "Attention may be given to both locations simultaneously, or attention may be switched rapidly back and forth between the two locations on a single trial" (p. 281). This is opposed to "switching" models, in which only one location is attended per trial. Shaw (1982), Sperling (1984), and Sperling and Melchner (1978a, 1978b) also defined trial-to-trial switching models as one class, including within-trial switching and simultaneous sharing in a second class. Obviously, this is a broader definition of sharing than we have in mind, since we wish to distinguish within-trial switching from simultaneous sharing. Other researchers have not always been as explicit as Shaw about including within-trial switching models together with sharing models, but it is clear that the predictions they derived for switching models hold only for trial-to-trial switching, and not for within-trial switching. In essence, then, these previous studies addressed the issue of whether attention can obtain information from more than one location on a given trial-not whether it can do so within a given brief interval of time.

4. A negative contingency could be introduced if the division of attention varied randomly from trial to trial, but we assume that this effect is small enough to ignore, at least with practiced observers.

5 . Contingency could be zero in one particular stochastic singleswitch model, in which there was trial-to-trial variation in the starting side but not in $d_{a}$. Specifically, if the subject set $d_{a}$ equal to $D / 2$, each side would receive the same amount of attended processing on all trials, and the complementary relationship would be eliminated. With $D$ varying randomly, however, the subject cannot choose a value of $d_{a}$ that is equal to $D / 2$ for more than one of the stimulus durations, so even this model predicts a negative contingency for at least three of the four stimulus durations tested.

6 . There will be no variation if the stimulus duration is an integer multiple of $2 \times d_{a}$ and $d_{a}$ is constant, even if the first side and the moment of first switch vary across trials. Under these conditions, each side is attended for exactly half of the stimulus duration, regardless of which side is attended first or the moment of the first switch. We will ignore this point here (but not in the subsequent simulations), because in this experiment the stimulus durations were not all integer multiples of any duration larger than $10 \mathrm{msec}$.

7. This prediction depends on the assumption-clearly supported by the results of the present experiment--that attention facilitates accurate responding.

8. If fixation did vary from trial to trial, this variation would cause negative contingencies like those predicted by switching models. When fixation drifted toward one side, sensitivity would be higher on that side and correspondingly lower on the other (i.e., negative contingency).

9. The literature contains little guidance on how to compute the predicted mean (i.e., expected value) and variance of sample $d$ 's from a given true $d_{m}^{\prime}$ specified by a model. Gourevitch and Galanter (1967) suggested one approximation for the variance, but our informal simulations indicated that it is quite inaccurate when $d^{\prime}$ is large. In the present computations, then, a brute-force numerical procedure was used to compute the predicted mean and variance from $d^{\prime} m$. The five-step procedure was based on the familiar formula $d^{\prime}=z$ (hit) $-z$ (false alarm), where $z$ is the inverse of the cumulative normal distribution function computed on the observed probability of a hit (Macmillan \& Creelman, 1991, Equation 1.3). (1) The criterion was assumed to be unbiased, and hence was set to $d_{m}^{\prime} / 2$. (2) The true hit and false-alarm prob- 
abilities, $p_{\mathrm{H}}$ and $p_{\mathrm{F}}$, were computed from the location of the criterion, assuming equal variance signal and noise distributions with means of $d_{m}^{\prime}$ and zero, respectively. (3) The number of possible hits in a sample of $N$ trials from the signal distribution, $n_{\mathrm{H}}$, is an integer value in the range $0-N$, and the probability of each value is given by the binomial distribution with parameters of $N$ and $p_{\mathrm{H}}$. Each value of $n_{\mathrm{H}}$ was converted to a possible value of $z($ hit $)$, namely, $z\left(n_{\mathrm{H}} / N\right)$. Note that the discrete probability distribution of $z$ (hit) is uniquely determined by this transformation of $n_{\mathrm{H}}$ values to $z$ (hit) values, so the expected value and variance of $z$ (hit) can be computed numerically. (4) Step 3 could be repeated, replacing $p_{\mathrm{H}}$ with $p_{\mathrm{F}}$ to get the mean and variance of $z$ (false alarm), but, in fact, this step was unnecessary. Because of the assumption of an unbiased criterion, $E[z$ (false alarm) $=-E[z($ hit $)]$ and $\operatorname{Var}\left[z(\right.$ false alarm $)=\operatorname{Var}[z($ hit $)]$. (5) The mean of $d^{\prime}$ is the difference of the expected values $E[z$ (hit $)]-E[z$ (false alarm) $)$, and the variance of $d^{\prime}$ is the sum of the corresponding variances, $\operatorname{Var}[z(\mathrm{hit})]+$ $\operatorname{Var}[z$ (false alarm) $]$.

10. Twelve of these were obtained with high discriminability, and 4 were obtained with medium; 11 were obtained at the two longest durations, and 5 were obtained at the two shortest; 9 were obtained in the $50 / 50$ attentional condition, and 7 were obtained in the $20 / 80$ and $80 / 20$ conditions.

11. Since we failed to reject the null hypothesis of zero contingency between left- and right-side performance, we conducted simulations of the switching models to see whether the experiment had adequate power. Each model was simulated under conditions corresponding to the testing of a single subject (e.g., 72 trials per condition). Simulations were restricted to the conditions with $50 \%$ of attention allocated to each side, because these require the fewest assumptions. For each simulated subject, the responses generated by the model were used to compute a chi-square value to test the hypothesis of independent response accuracy on the two sides.

The results indicated that power was excellent. For the trial-to-trial switching model, it was virtually certain that a significant negative contingency would have been obtained in the experiment (i.e., power essentially equal to one). Three versions of the stochastic single-switch model were simulated. The distribution of $d_{a}$ across trials was (1) con- stant, (2) normal, or (3) uniform, and there was wide variation across simulations of the parameter(s) of the distribution of $d_{a}$. For all versions of the stochastic single-switch model, power exceeded .9. In simulations of stochastic multiple-switch models, attention switched sides every $d_{a}$ msec until stimulus offset; $d_{a}$ varied from 5 to $500 \mathrm{msec}$ in different sets of simulations, and power depended on the assumed switching rate. With switches no faster than once per $80 \mathrm{msec}$, power was at least .97 . With switches every 60,40 , or $20 \mathrm{msec}$, power dropped to $.85, .69$, and .49 , respectively. There was little effect of whether the all-or-none device was assumed to be "synchronized," making its first switch exactly $d_{a}$ msec after stimulus onset, or "unsynchronized," making its first switch at a random moment after stimulus onset.

Two additional attempts were made to reconcile stochastic singleand multiple-switch models with the lack of negative contingencies by including in the model a source of positive contingency to counteract the negative contingency otherwise predicted. In one attempt, the subjects randomly guessed for both responses on some trials. Power was virtually undiminished with $2 \%$ guesses and was quite high even with $5 \%$ guesses. Because the response accuracies generated by the simulations with $5 \%$ guesses were far below the observed levels, it was not reasonable to try larger percentages of guesses. In the other attempt, random trial-to-trial fluctuations in sensitivity were introduced, increasing or decreasing $d^{\prime}$ for both tasks by up to $50 \%$ on a given trial. Again, these simulations revealed negligible reductions in power.

12. Details available on request.

13. In fact, these models must actually predict performance to be slightly worse than $75 \%$ correct, because responses were not perfect in the single-task conditions. Since performance is significantly better than $75 \%$ and the models can be rejected, we will not try to establish just how much lower than $75 \%$ the models actually predict accuracy to be.

(Manuscript received January 11, 1993; revision accepted for publication April 11, 1994.) 